12

\title{
Черенковское излучение, генерируемое электронным сгустком в прямоугольном диэлектрическом волноводе с трансверсально-изотропным заполнением
}

\author{
(С) И.Л. Шейнман, Ю.С. Шейнман
}

Санкт-Петербургский государственный электротехнический университет, 197376 Санкт-Петербург, Россия

e-mail: ishejnman@yandex.ru

Поступило в Редакцию 26 июня 2017 г.

В окончательной редакции 26 июня 2017 г.

Принято к публикации 17 марта 2019 г.

Диэлектрические волноводы интенсивно исследуются в качестве ускорительных структур, возбуждаемых электронным пучком. Прямоугольные диэлектрические структуры используются как для проверки принципов новых ускорительных схем, так и для изучения электрических свойств материалов заполнения. Ряд диэлектрических материалов, используемых в качестве заполнения волноводов, имеют анизотропию свойств (сапфир, керамические пленки). Анизотропия может оказывать значительное влияние на кильватерные поля, генерируемые электронным пучком в структуре. Проведен аналитический расчет излучения Вавилова-Черенкова, генерируемого релятивистским электронным сгустком в прямоугольном волноводе с поперечно-неоднородным трансверсально-изотропным диэлектрическим заполнением. Представлен метод построения ортогонального базиса поперечного оператора с последующим его использованием для нахождения кильватерного поля. Получены дисперсионное уравнение для структуры и выражения для кильватерного поля, создаваемого точечным электронным сгустком в трансверсально-изотропной прямоугольной диэлектрической структуре. На основе изложенного формализма проведены расчеты параметров ускорительной структуры на основе сапфира, допускающей генерацию полей свыше $100 \mathrm{MV} / \mathrm{m}$.

Ключевые слова: Кильватерное ускорение, ускорительная структура, прямоугольный волновод, диэлектрический волновод, электронный сгусток.

DOI: $10.21883 /$ JTF.2019.09.48073.2408

\section{Введение}

Эксперименты в области физики высоких энергий требуют разработки новых технологий для создания более совершенных ускорителей. Перспективным направлением в разработке линейных коллайдеров является развитие методов кильватерного ускорения в структурах с диэлектрическим заполнением [1,2].

Ускоряющей структурой с диэлектрическим заполнением является заключенный в проводящую металлическую оболочку диэлектрический волновод с осевым вакуумным каналом для пролета пучка. Для ускорения интенсивного электронного сгустка высоких энергий в волноводе возбуждают излучение Вавилова-Черенкова (кильватерное поле). Кильватерное поле может быть создано сильноточным, коротким низкоэнергетическим электронным пучком, называемым ведущим сгустком или драйвером. Ускоряемый пучок должен двигаться за драйвером на определенном расстоянии, чтобы все время находиться в ускоряющей фазе поля кильватерной СВЧ-волны.

Применение диэлектрических кильватерных структур позволяет не только существенно увеличить энергию ускоряемых частиц на небольшом расстоянии, но и управлять частотным спектром структуры посредством введения дополнительных сегнетоэлектрических слоев [3]. Для создания ускорительной структуры представляет особый интерес использование новых перспективных материалов, обладающих уникальными свойствами, например, алмаза, сапфира [4], тонких керамических пленок.

Как правило, при создании структур с диэлектрическим заполнением используют цилиндрическую геометрию, что оказывается важным для достижения максимальных ускоряющих градиентов и получения максимально возможного шунтового импеданса структуры. Аналитический модовый анализ подобных ускоряющих структур для продольных и поперечных компонент электрического поля был разработан в [5]. В последние десятилетия разрабатывались методы моделирования структур с диэлектрическим заполнением, обладающих прямоугольным сечением [6-11].

Внимание к прямоугольным диэлектрическим структурам вызвано как существующими технологическими сложностями изготовления цилиндрических структур, а именно высокими требованиями к допускам на геометрические параметры и однородность диэлектрической проницаемости заполнения вдоль структур [3], так и возможным использованием для генерации ленточного электронного сгустка.

Прямоугольные структуры могут быть использованы для тестовых экспериментов при анализе новых 
ускорительных схем [12] и для изучения свойств материалов, перспективных для высоких темпов ускорения и импульсного нагрева структуры (сапфир, керамические пленки) [4]. Преимущество прямоугольных структур заключается в простоте технологии изготовления. В частотном диапазоне $0.5-1.0 \mathrm{THz}$ прямоугольные структуры рассматриваются наравне с цилиндрическими [4]. В THz-диапазоне прямоугольная геометрия может оказаться предпочтительной из-за трудностей с высокоточным изготовлением структур цилиндрической геометрии.

Теоретический анализ диэлектрических ускорительных структур прямоугольной геометрии к настоящему времени проводился в ряде работ [6-17]. Для нахождения амплитуд мод излучения Вавилова-Черенкова, возбуждаемых в прямоугольном волноводе с диэлектрическим заполнением, ранее использовались техника согласования импедансов [7-9], наряду с методом поперечного резонанса [11]. При использовании подобного формализма вместо прямого решения неоднородной системы уравнений Максвелла (что является стандартным аналитическим подходом при анализе кильватерных полей в цилиндрических структурах [5]) приходится выражать амплитуды кильватерных полей через шунтовой импеданс (или интегральный фактор потерь) каждой из мод структуры. Подобный подход использует определенные приближения, тогда как прямое решение неоднородной системы уравнений Максвелла без косвенных построений всегда предпочтительнее для анализа задач генерации в волноведущих структурах. В работах $[10,12]$ приведен анализ на основе прямого решения уравнений Максвелла двухканальной прямоугольной структуры с диэлектрическим заполнением, разрабатываемой для повышения коэффициента трансформации энергии от ведущего пучка к ускоряемому пучку. Однако ряд использованных в [12] математических моментов (таких, как изменение знака весового коэффициента в определении нормы и полнота рассматриваемой системы функций, по которой велось разложение) остался без строгого обоснования и требует дополнительного исследования.

В $[13,14,17]$ разработана строгая теория возбуждения релятивистским пучком прямоугольного волновода с изотропным заполнением. Мы использовали аналогичный метод, но с рядом улучшений при нахождении тангенциальных компонент полей.

\section{Основные уравнения для нахождения кильватерных полей в волноводе}

Для вывода выражений для кильватерных полей воспользуемся системой уравнений Максвелла в системе СИ (1), дополненных материальными соотношениями (2):

$$
\begin{array}{cl}
\nabla \times \mathbf{E}=-\frac{\partial \mathbf{B}}{\partial t}, & \nabla \times \mathbf{H}=\frac{\partial D}{\partial t}-e n \mathbf{v} \\
\nabla \mathbf{B}=0, & \nabla \mathbf{D}=-e n
\end{array}
$$

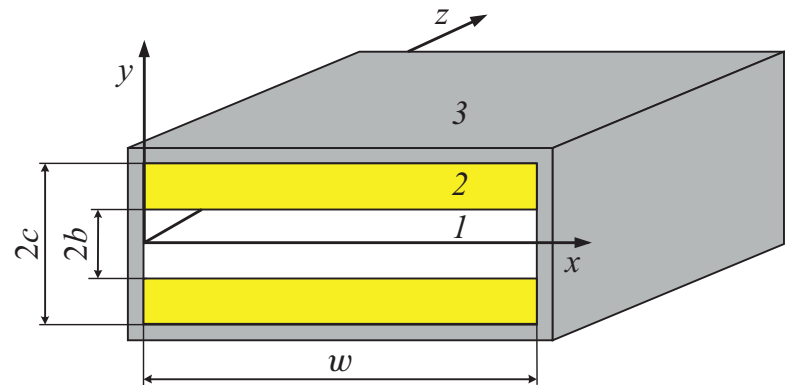

Рис. 1. Кильватерная структура на основе прямоугольного диэлектрического волновода.

$$
\mathbf{B}=\mu_{0} \hat{\mu} \mathbf{H}, \quad \mathbf{D}=\varepsilon_{0} \hat{\varepsilon} \mathbf{E},
$$

где $\mathbf{E}, \mathbf{H}$ - векторы напряженности; D, B - индукции электрического и магнитного полей; $\mathbf{v}-$ скорость движения заряженных частиц (электронного сгустка); $n$ концентрация электронов в сгустке; $e$ - элементарный заряд; $t$ - время.

Сгусток электронов движется в вакуумном канале вдоль оси волновода. Кильватерное поле создается за сгустком, когда материал диэлектрика удовлетворяет условию излучения Черенкова $v=\beta c$ и $\beta>\varepsilon^{-1 / 2}$, где $c$ - скорость электромагнитной волны в вакууме; $v-$ скорость движения заряженных частиц (пучка), $\beta=v / c$.

Концентрация точечного заряда $q$, движущегося параллельно продольной оси $z$ волновода, записывается следующим образом:

$$
n(x, y, z, t)=\frac{q}{-e} \delta\left(z-v t-z_{0}\right) \delta\left(x-x_{0}\right) \delta\left(y-y_{0}\right),
$$

где $x, y$ - поперечные координаты; $z$ - продольная координата, направленная по оси волновода; $x_{0}, y_{0}, z_{0}-$ координаты заряда в начальный момент времени.

Рассмотрим прямоугольный волновод с симметричным заполнением в виде трансверсально-изотропных диэлектрических слоев, параллельных оси $x$, и вакуумным каналом в центре (рис. 1). В этом случае в направлении оси $y$ заполнение является неоднородным и компоненты тензоров диэлектрической и магнитной проницаемости зависят от $y: \hat{\varepsilon}=\hat{\varepsilon}(y), \hat{\mu}=\hat{\mu}(y)$ :

$$
\begin{aligned}
& \hat{\varepsilon}=\left(\begin{array}{ccc}
\varepsilon_{\|}(y) & 0 & 0 \\
0 & \varepsilon_{\perp}(y) & 0 \\
0 & 0 & \varepsilon_{\|}(y)
\end{array}\right), \\
& \hat{\mu}=\left(\begin{array}{ccc}
\mu_{\|}(y) & 0 & 0 \\
0 & \mu_{\perp}(y) & 0 \\
0 & 0 & \mu_{\|}(y)
\end{array}\right) .
\end{aligned}
$$

Преобразуем исходные уравнения Максвелла с учетом материальных соотношений для этого случая. Находя из последних двух уравнений системы (1) дивергенции векторов напряженности электрического и 
магнитного полей, будем иметь

$$
\begin{gathered}
\nabla \cdot \mathbf{E}=-\frac{1}{\varepsilon_{0} \varepsilon_{\|}}\left(e n+\varepsilon_{0}\left(\varepsilon_{\perp}-\varepsilon_{\|}\right) \frac{\partial E_{y}}{\partial y}+\varepsilon_{0} E_{y} \frac{\partial \varepsilon_{\perp}}{\partial y}\right), \\
\nabla \cdot \mathbf{H}=-\left(\frac{\mu_{\perp}-\mu_{\|}}{\mu_{\|}} \frac{\partial H_{y}}{\partial y}+\frac{H_{y}}{\mu_{\|}} \frac{\partial \mu_{\perp}}{\partial y}\right) .
\end{gathered}
$$

Применяя оператор ротора к первому уравнению Максвелла (1) и используя второе уравнение, получим для поперечной компоненты поля $E_{y}$

$$
\begin{aligned}
& \left(\nabla^{2} E_{y}-\frac{\varepsilon_{\perp} \mu_{\|}}{c^{2}} \frac{\partial^{2} E_{y}}{\partial t^{2}}\right) \\
& =-\frac{\partial}{\partial y}\left(-\frac{e n}{\varepsilon_{0} \varepsilon_{\|}}\right)-\frac{\partial}{\partial y}\left(\frac{\varepsilon_{\perp}-\varepsilon_{\|}}{\varepsilon_{\|}} \frac{\partial E_{y}}{\partial y}\right)-\frac{\partial}{\partial y}\left(\frac{E_{y}}{\varepsilon_{\|}} \frac{\partial \varepsilon_{\perp}}{\partial y}\right) .
\end{aligned}
$$

Применяя оператор ротора ко второму уравнению Максвелла (1) и учитывая (5), получим уравнение для нормальной к диэлектрическому слою компоненты поля $H_{y}$

$$
\begin{aligned}
\left(\Delta H_{y}-\frac{\varepsilon_{\|} \mu_{\perp}}{c^{2}} \frac{\partial^{2} H_{y}}{\partial t^{2}}\right)=e(\nabla \times(n \mathbf{v}))_{y} \\
\quad-\frac{\partial}{\partial y}\left(\frac{\mu_{\perp}-\mu_{\|}}{\mu_{\|}} \frac{\partial H_{y}}{\partial y}\right)-\frac{\partial}{\partial y}\left(\frac{H_{y}}{\mu_{\|}} \frac{\partial \mu_{\perp}}{\partial y}\right) .
\end{aligned}
$$

Учитывая 3, будем искать решения в виде $E_{y}(x, y, z, t)=E_{y}(x, y, z-v t)=E_{y}(x, y, \xi), H_{y}(x, y, z, t)=$ $=H_{y}(x, y, z-v t)=H_{y}(x, y, \xi)$, где $\xi=z-v t$. Определим дифференциальные операторы

$$
\begin{aligned}
& \hat{T}_{E} E_{y}=\frac{1}{\left(1-\varepsilon_{\perp} \mu_{\|} \beta^{2}\right)}\left[\frac{\partial^{2} E_{y}}{\partial x^{2}}+\frac{\partial}{\partial y}\left(\frac{1}{\varepsilon_{\|}} \frac{\partial}{\partial y}\left[\varepsilon_{\perp} E_{y}\right]\right)\right], \\
& \hat{T}_{H} E_{y}=\frac{1}{\left(1-\varepsilon_{\|} \mu_{\perp} \beta^{2}\right)}\left[\frac{\partial^{2} H_{y}}{\partial x^{2}}+\frac{\partial}{\partial y}\left(\frac{1}{\mu_{\|}} \frac{\partial\left(\mu_{\perp} H_{y}\right)}{\partial y}\right)\right] .
\end{aligned}
$$

Тогда получим уравнения Гельмгольца

$$
\begin{gathered}
\frac{\partial^{2} E_{y}}{\partial \xi^{2}}+\hat{T}_{E} E_{y}=\frac{-e}{\varepsilon_{0}\left(1-\varepsilon_{\perp} \mu_{\|} \beta^{2}\right)} \frac{\partial}{\partial y}\left(\frac{n}{\varepsilon_{\|}}\right), \\
\frac{\partial^{2} H_{y}}{\partial \xi^{2}}+\hat{T}_{H} H_{y}=\frac{-e v}{1-\varepsilon_{\|} \mu_{\perp} \beta^{2}}\left(\frac{\partial n}{\partial x}\right) .
\end{gathered}
$$

Полученные не зависящие друг от друга уравнения (8) и (9) описывают возбуждение LM- и LE-мод волновода соответственно.

\section{Собственные функции поперечных операторов}

Из системы уравнений Максвелла следуют граничные условия на напряженности электрического и магнитного полей:

$$
\left.E_{y}\right|_{x=0}=0 ;\left.\quad E_{y}\right|_{x=w}=0 ;\left.\quad \frac{\partial E_{y}}{\partial y}\right|_{y= \pm c}=0 ;
$$

$$
\left.\frac{\partial H_{y}}{\partial x}\right|_{x=0}=0 ;\left.\quad \frac{\partial H_{y}}{\partial x}\right|_{x=w}=0 ;\left.\quad H_{y}\right|_{y= \pm c}=0
$$

Рассмотрим задачи на собственные функции $\Psi(x, y)$ операторов $\hat{T}_{E}$ и $\hat{T}_{H}$ :

$$
\begin{gathered}
\hat{T}_{E} \Psi_{E}(x, y)=\lambda_{E} \Psi_{E}(x, y), \\
\Psi_{E}(0, y)=0, \quad \Psi_{E}(w, y)=0, \\
\left.\frac{\partial \Psi_{E}(x, y)}{\partial y}\right|_{y=c}=0,\left.\quad \frac{\partial \Psi_{E}(x, y)}{\partial y}\right|_{y=-c}=0 ; \\
\hat{T} \Psi_{H}(x, y)=\lambda_{H} \Psi_{H}(x, y), \\
\left.\frac{\partial \Psi_{H}(x, y)}{\partial x}\right|_{x=0}=0 ;\left.\quad \frac{\partial \Psi_{H}(x, y)}{\partial x}\right|_{x=w}=0 ; \\
\Psi_{H}(x,-c)=0, \quad \Psi_{H}(x, c)=0 .
\end{gathered}
$$

Рассмотрим задачу на собственные функции

$$
\begin{gathered}
\frac{\partial^{2}}{\partial x^{2}} X_{n}(x)=-k_{x}^{2} X_{n}(x), \quad X_{E}(0)=0, \quad X_{E}(w)=0, \\
\frac{\partial X_{H}(0)}{\partial x}=0, \quad \frac{\partial X_{H}(w)}{\partial x}=0 .
\end{gathered}
$$

Решения задачи образуют ортогональные базисы вида

$$
X_{E n}(x)=\sin k_{x} x, X_{H n}(x)=\cos k_{x} x, k_{x}=\pi n / w .
$$

Разложим $\Psi(x, y)$ в ряд по собственным функциям задачи (12):

$$
\Psi(x, y)=\sum_{n=1}^{\infty} X_{n}(x) Y(y)
$$

Подставляя (14) в (10) и (11), получим

$$
\begin{aligned}
& \frac{1}{\left(1-\varepsilon_{\perp} \mu_{\|} \beta^{2}\right)} \frac{\partial}{\partial y}\left(\frac{1}{\varepsilon_{\|}} \frac{\partial\left(\varepsilon_{\perp} Y_{E_{y}}(y)\right)}{\partial y}\right) \frac{k_{x}^{2}}{\left(1-\varepsilon_{\perp} \mu_{\|} \beta^{2}\right)} Y_{E_{y}}(y)=\lambda_{E} Y_{E_{y}}(y), \\
& \frac{\partial Y_{E_{y}}( \pm c)}{\partial y}=0 \\
& \frac{1}{\left(1-\varepsilon_{\|} \mu_{\perp} \beta^{2}\right)} \frac{\partial}{\partial y}\left(\frac{1}{\mu_{\|}} \frac{\partial\left(\mu_{\perp} Y_{H_{y}}(y)\right)}{\partial y}\right) \\
&-\frac{k_{x}^{2}}{\left(1-\varepsilon_{\|} \mu_{\perp} \beta^{2}\right)} Y_{H_{y}}(y)=\lambda_{H} Y_{H_{y}}(y), \\
& Y_{H_{y}}( \pm c)=0 .
\end{aligned}
$$

Решения (15), (16) для каждой из областей имеют вид

$$
\begin{gathered}
Y_{E_{y}}(y)=C_{E} \sin k_{y E} y+D_{E} \cos k_{y E} y, \\
Y_{H_{y}}(y)=C_{H} \sin k_{y H} y+D_{H} \cos k_{y H} y,
\end{gathered}
$$


где

$$
\begin{aligned}
& k_{y 2 E}=\sqrt{\frac{\varepsilon_{\| 2}}{\varepsilon_{\perp 2}}\left[\left(\varepsilon_{\perp 2} \mu_{\| 2} \beta^{2}-1\right) \lambda_{E}-k_{x}^{2}\right]}, \\
& k_{y 2 H}=\sqrt{\frac{\mu_{\| 2}}{\mu_{\perp 2}}\left[\left(\varepsilon_{\| 2} \mu_{\perp 2} \beta^{2}-1\right) \lambda_{H}-k_{x}^{2}\right]}
\end{aligned}
$$

для диэлектрической среды. Для вакуумного канала, учитывая что $\left(\varepsilon_{1} \mu_{1} \beta^{2}-1\right)=\left(\beta^{2}-1\right)<0$, необходимо произвести замену $k_{y 1} \rightarrow i k_{y}: k_{y 1}=\sqrt{k_{x}^{2}+\lambda_{E}\left(1-\beta^{2}\right)} \mathrm{c}$ соответствующей заменой тригонометрической функции на гиперболическую.

Используя граничные условия и условия непрерывности $D_{y}$ и $B_{y}$ при $y= \pm b$, получим, что собственные функции $Y_{E_{y}}(y)$ и $Y_{H_{y}}(y)$ распадаются на систему симметричных и антисимметричных мод относительно оси волновода. В итоге для симметричных относительно оси волновода мод получим

$$
\begin{aligned}
& Y_{E_{y}}(y)=A_{E}\left\{\begin{array}{l}
\frac{1}{\varepsilon_{\perp 2}} \cos \left(k_{y 2 E}(c-y)\right), \\
{\left[\begin{array}{l}
\mathrm{ch} \\
\mathrm{sh}
\end{array}\right]\left(k_{y 1} y\right) \frac{\cos \left(k_{y 2 E}(c-b)\right)}{\left[\begin{array}{c}
\mathrm{ch} \\
\mathrm{sh}
\end{array}\right]\left(k_{y 1} b\right)}} \\
{[ \pm] \frac{1}{\varepsilon_{\perp 2}} \cos \left(k_{y 2 E}(c+y)\right) .}
\end{array}\right. \\
& Y_{H_{y}}(y)=A_{H}\left\{\begin{array}{l}
\frac{1}{\mu_{\perp 2}} \sin \left(k_{y 2 H}(c-y)\right), b \leq y \leq c ; \\
{\left[\begin{array}{l}
\mathrm{ch} \\
\mathrm{sh}
\end{array}\right]\left(k_{y 1} y\right) \frac{\sin \left(k_{y 2 H}(c-b)\right)}{\left[\begin{array}{l}
\mathrm{ch} \\
\mathrm{sh}
\end{array}\right]\left(k_{y 1} b\right)},-b \leq y \leq b ;} \\
{[ \pm] \frac{1}{\mu_{\perp 2}} \sin \left(k_{y 2 H}(c+y)\right),-c \leq y \leq-b .}
\end{array}\right.
\end{aligned}
$$

Здесь в обозначениях $[\cdot]$ верхняя операция соответствует симметричной моде, нижняя - антисимметричной. Из условий нормировки $\left\langle\Phi_{E}, \Psi_{E}\right\rangle=1$ и $\left\langle\Phi_{H}, \Psi_{H}\right\rangle=1$ (см. ниже) найдем нормирующие коэффициенты:

$$
\begin{aligned}
A_{E} & =\sqrt{\frac{2}{w}}\left(\left(1-\beta^{2}\right) \frac{\cos ^{2}\left(k_{y 2 E}(c-b)\right)}{\varepsilon_{1}\left(\left[\begin{array}{l}
\mathrm{ch} \\
\mathrm{sh}
\end{array}\right]\left(k_{y 1} b\right)\right)^{2}}\left(\frac{\operatorname{sh}\left(2 k_{y 1} b\right)}{2 k_{y 1}}[ \pm] b\right)\right. \\
& \left.+\frac{\left(1-\varepsilon_{\perp 2} \mu_{\| 2} \beta^{2}\right)}{\varepsilon_{\perp 2}}\left(c-b+\frac{\sin \left(2 k_{y 2 E}(c-b)\right)}{2 k_{y 2 E}}\right)\right)^{-\frac{1}{2}}, \\
A_{H} & =\sqrt{\frac{2}{w}}\left(\left(1-\beta^{2}\right) \frac{\sin ^{2}\left(k_{y 2 E}(c-b)\right)}{\mu_{1}\left(\left[\begin{array}{l}
\mathrm{ch} \\
\mathrm{sh}
\end{array}\right]\left(k_{y 1} b\right)\right)^{2}}\left(\frac{\operatorname{sh}\left(2 k_{y 1} b\right)}{2 k_{y 1}}[ \pm] b\right)\right. \\
& \left.+\frac{\left(1-\varepsilon_{\| 2} \mu_{\perp 2} \beta^{2}\right)}{\mu_{\perp 2}}\left(c-b-\frac{\sin \left(2 k_{y 2 E}(c-b)\right)}{2 k_{y 2 E}}\right)\right)^{-\frac{1}{2}} .
\end{aligned}
$$

Из однородных уравнений Максвелла может быть получено выражение для продольной компоненты напряженности электрического поля

$$
E_{z}=\frac{1}{\varepsilon_{\|}\left(\lambda+k_{x}^{2}\right)} \frac{\partial^{2}\left(\varepsilon_{\perp} E_{y}\right)}{\partial \xi \partial y}
$$

Из непрерывности $E_{z}$ на границе диэлектрика с вакуумным каналом следует непрерывность $\frac{\varepsilon_{\perp}}{\varepsilon_{\|}} \frac{\partial Y_{E}}{\partial y}$, откуда получим дисперсионное уравнение для симметричных относительно оси волновода LM-мод:

$$
\varepsilon_{\| 2} k_{y 1} \operatorname{th}\left(k_{y 1} b\right)-\varepsilon_{1} k_{y 2 E} \operatorname{tg}\left(k_{y 2 E}(c-b)\right)=0 .
$$

Системе антисимметричных LM-мод соответствует дисперсионное уравнение

$$
\varepsilon_{\| 2} k_{y 1} \operatorname{cth}\left(k_{y 1} b\right)-\varepsilon_{1} k_{y 2 E} \operatorname{tg}\left(k_{y 2 E}(c-b)\right)=0 .
$$

Из непрерывности

$$
H_{z}=\frac{\mu_{\perp}}{\mu_{\|}\left(\lambda+k_{x}^{2}\right)} \frac{\partial^{2} H_{y}}{\partial y \partial \xi}
$$

на границе диэлектрика с вакуумным каналом получим дисперсионное уравнение для симметричных LE-мод

$$
\mu_{\| 2} k_{y 1} \operatorname{th}\left(k_{y 1} b\right)+\mu_{1} k_{y 2 H} \operatorname{ctg}\left(k_{y 2 H}(c-b)\right)=0 .
$$

Системе антисимметричных LE-мод соответствует дисперсионное уравнение

$$
\mu_{1} k_{y 2 H} \operatorname{th}\left(k_{y 1} b\right)+\mu_{\| 2} k_{y 1} \operatorname{tg}\left(k_{y 2 H}(c-b)\right)=0 .
$$

В случае изотропной среды $\varepsilon_{2 \perp}=\varepsilon_{2 \|}=\varepsilon_{2}$, $\mu_{2 \|}=\mu_{2 \perp}=\mu_{2}$ дисперсионные уравнения совпадают с уравнениями, полученными в [14].

\section{Представление решения в виде разложения по собственным функциям поперечных операторов}

Рассмотрим подробнее оператор, стоящий в левой части уравнения (15). Для его собственных функций в пространстве $L_{2}\left([-c, c],\left|\varepsilon_{\perp}\left(1-\varepsilon_{\perp} \mu_{\|} \beta^{2}\right)\right|\right)$, где $\rho=\left|\varepsilon_{\perp}\left(1-\varepsilon_{\perp} \mu_{\|} \beta^{2}\right)\right|-$ вес, скалярное произведение будет иметь вид

$$
\left\langle\varphi_{E}, \psi_{E}\right\rangle=\int_{-c}^{c}\left|\varepsilon_{\perp}\left(1-\varepsilon_{\perp} \mu_{\| \beta^{2}}\right)\right| \varphi_{E}^{*} \psi_{E} d y
$$

где $\varphi$ и $\psi \in L_{2}([-c, c],|\rho|)$,

$$
\begin{aligned}
\hat{T}_{E_{y}} Y_{E}(x)= & \frac{1}{1-\varepsilon_{\perp} \mu_{\|} \beta^{2}} \frac{\partial}{\partial y}\left(\frac{1}{\varepsilon_{\|}} \frac{\partial \varepsilon_{\perp} Y_{E_{y}}(x)}{\partial y}\right) \\
& -\frac{k_{x}^{2} Y_{E_{y}}(Y)}{1-\varepsilon_{\perp} \mu_{\|} \beta^{2}},
\end{aligned}
$$




$$
\begin{aligned}
\left\langle\varphi, \hat{T}_{E_{y}} \psi\right\rangle & =\int_{-c}^{c} \operatorname{sign}\left(1-\varepsilon_{\perp} \mu_{\|} \beta^{2}\right) \\
& \times\left[\bar{\varphi}\left|\varepsilon_{\perp}\right|\left[\frac{\partial}{\partial y}\left(\frac{1}{\varepsilon_{\|}} \frac{\partial \varepsilon_{\perp} \psi}{\partial y}\right)\right]-k_{x}^{2}\left|\varepsilon_{\perp}\right| \bar{\varphi} \psi\right] d y .
\end{aligned}
$$

Используя интегрирование по частям, получим

$$
\begin{aligned}
\left\langle\varphi, T_{E_{y}} \psi\right\rangle & =\left[\operatorname { s i g n } ( 1 - \varepsilon _ { \perp } \mu _ { \| } \beta ^ { 2 } ) \frac { | \varepsilon _ { \perp } | } { \varepsilon _ { \| } } \left(\bar{\varphi} \frac{\partial \varepsilon_{\perp} \psi}{\partial y}\right.\right. \\
& \left.\left.-\psi\left[\frac{\partial \varepsilon_{\perp} \bar{\varphi}}{\partial y}\right]\right)\right]\left.\right|_{-c} ^{c}+\int_{-c}^{c} \operatorname{sign}\left(1-\varepsilon_{\perp} \mu_{\|} \beta^{2}\right) \\
& \times\left[\left|\varepsilon_{\perp}\right| \overline{\frac{\partial}{\partial y}} \frac{1}{\varepsilon_{\|}} \frac{\partial \varepsilon_{\perp} \varphi}{\partial y} \psi-k_{x}^{2}\left|\varepsilon_{\perp}\right| \bar{\varphi} \psi\right] d y .
\end{aligned}
$$

Сопряженный дифференциальный оператор $\hat{T}_{E_{v}}^{*}$ может быть построен на основе соотношения

$$
\left\langle\varphi, \hat{T}_{E_{y}} \psi\right\rangle-\left\langle\hat{T}_{E_{y}}^{*} \varphi, \psi\right\rangle=0 .
$$

Его дифференциальное выражение совпадает с исходным оператором, однако собственные функции задачи отличаются от собственных функций исходного оператора граничными условиями.

Производя дифференцирование, получим

$$
\begin{aligned}
& \left\langle\varphi, \hat{T}_{E_{y}}, \psi\right\rangle-\left\langle\hat{T}_{E_{y}} \varphi, \psi\right\rangle=\left[\operatorname{sign}\left(1-\varepsilon_{\perp} \mu_{\|} \beta^{2}\right) \frac{\left|\varepsilon_{\perp}\right|}{\varepsilon_{\|}}\right. \\
& \left.\times\left(\bar{\varphi} \psi \frac{\partial \varepsilon_{\perp}}{\partial y}+\varepsilon_{\perp} \bar{\varphi} \frac{\partial \psi}{\partial y}-\bar{\varphi} \psi \frac{\partial \varepsilon_{\perp}}{\partial y}-\varepsilon_{\perp} \psi \frac{\partial \bar{\varphi}}{\partial y}\right)\right]\left.\right|_{-c} ^{c} \\
& =-\left.\left|\varepsilon_{\perp}\right| \frac{\varepsilon_{\perp}}{\varepsilon_{\|}} \bar{\varphi} \frac{\partial \psi}{\partial y}\right|_{y=-c}+\left.\left|\varepsilon_{\perp}\right| \frac{\varepsilon_{\perp}}{\varepsilon_{\|}} \bar{\varphi} \frac{\partial \psi}{\partial y}\right|_{y=c} \\
& +\left(\left.\left|\varepsilon_{\perp}\right| \frac{\varepsilon_{\perp}}{\varepsilon_{\|}} \bar{\varphi} \frac{\partial \psi}{\partial y}\right|_{y=-b-0}+\left.\left|\varepsilon_{\perp}\right| \frac{\varepsilon_{\perp}}{\varepsilon_{\|}} \bar{\varphi} \frac{\partial \psi}{\partial y}\right|_{y=-b+0}\right) \\
& +\left(\left.\left|\varepsilon_{\perp}\right| \frac{\varepsilon_{\perp}}{\varepsilon_{\|}} \bar{\varphi} \frac{\partial \psi}{\partial y}\right|_{y=b-0}+\left.\left|\varepsilon_{\perp}\right| \frac{\varepsilon_{\perp}}{\varepsilon_{\|}} \bar{\varphi} \frac{\partial \psi}{\partial y}\right|_{y=b+0}\right) \\
& +\left[-\left.\left|\varepsilon_{\perp}\right| \frac{\varepsilon_{\perp}}{\varepsilon_{\|}} \psi \frac{\partial \bar{\varphi}}{\partial y}\right|_{y=-c}+\left.\left|\varepsilon_{\perp}\right| \frac{\varepsilon_{\perp}}{\varepsilon_{\|}} \psi \frac{\partial \bar{\varphi}}{\partial y}\right|_{y=c}\right. \\
& +\left(\left.\left|\varepsilon_{\perp}\right| \frac{\varepsilon_{\perp}}{\varepsilon_{\|}} \psi \frac{\partial \bar{\varphi}}{\partial y}\right|_{y=-b-0}+\left.\left|\varepsilon_{\perp}\right| \frac{\varepsilon_{\perp}}{\varepsilon_{\|}} \psi \frac{\partial \bar{\varphi}}{\partial y}\right|_{y=-b+0}\right) \\
& \left.+\left(\left.\left|\varepsilon_{\perp}\right| \frac{\varepsilon_{\perp}}{\varepsilon_{\|}} \psi \frac{\partial \bar{\phi}}{\partial y}\right|_{y=b-0}+\left.\left|\varepsilon_{\perp}\right| \frac{\varepsilon_{\perp}}{\varepsilon_{\|}} \psi \frac{\partial \bar{\varphi}}{\partial y}\right|_{y=b+0}\right)\right]
\end{aligned}
$$

Из непрерывности нормальных компонент индукции электрического поля $D_{y}$ на границе раздела диэлектрик-вакуум следует непрерывность произведений $\varepsilon_{\perp} \psi_{E}$. Из непрерывности тангенциальных компонент электрического поля $E_{z}$ и $E_{x}$ на границе раздела диэлектрик-вакуум следует непрерывность выражений $\frac{\varepsilon_{\perp}}{\varepsilon_{\|}} \frac{\partial \psi_{E}}{\partial y}$. Из граничных условий на верхней и нижней стенках волновода следует $\partial \psi_{E} /\left.\partial y\right|_{y= \pm c}=0$. Для удовлетворения (18) необходимо, чтобы собственные функции $\varphi$ сопряженного оператора $\hat{T}_{E_{y}}^{*}$ удовлетворяли граничным условиям:

$$
\begin{gathered}
\left|\varepsilon_{2 \perp}\right| \varphi_{E}(-b-0)=-\varepsilon_{1} \varphi_{E}(-b+0), \\
\varepsilon_{1} \varphi_{E}(b-0)=-\left|\varepsilon_{2 \perp}\right| \varphi_{E}(b+0), \\
\left.\frac{\partial \varphi_{E}}{\partial y}\right|_{y= \pm c}=0, \quad \frac{\left|\varepsilon_{2 \perp}\right|}{\varepsilon_{2 \|}} \frac{\partial \varphi_{E}(-b-0)}{\partial y}=-\frac{\partial \varphi_{E}(-b+0)}{\partial y}, \\
\frac{\partial \varphi_{E}(b-0)}{\partial y}=-\frac{\left|\varepsilon_{2 \perp}\right|}{\varepsilon_{2} \mid} \frac{\partial \varphi_{E}(b+0)}{\partial y} .
\end{gathered}
$$

Аналогично для собственных функций магнитного оператора (16) в пространстве $L_{2}\left([-c, c],\left|\mu_{\perp}\left(1-\varepsilon_{\|} \mu_{\perp} \beta^{2}\right)\right|\right)$ скалярное произведение будет

$$
\left\langle\varphi_{H}, \psi_{H}\right\rangle=\int_{-c}^{c}\left|\mu_{\perp}\left(1-\varepsilon_{\|} \mu_{\perp} \beta^{2}\right)\right| \varphi_{H}^{*} \psi_{H} d y,
$$

а граничные условия для сопряженного оператора $\hat{T}_{H_{y}}^{*}$

$$
\begin{gathered}
\left|\mu_{2 \perp}\right| \varphi_{H}(-b-0)=-\mu_{1} \varphi_{H}(-b+0) ; \\
\mu_{1} \varphi_{H}(b-0)=-\left|\mu_{2 \perp}\right| \varphi_{H}(b+0), \\
\left.\varphi_{H}\right|_{y= \pm c}=0 \quad \frac{\left|\mu_{2 \perp}\right|}{\mu_{2 \|}} \frac{\partial \varphi_{H}(-b-0)}{\partial y}=-\frac{\partial \varphi_{H}(-b+0)}{\partial y}, \\
\frac{\partial \varphi_{H}(-b+0)}{\partial y}=-\frac{\left|\mu_{2 \perp}\right|}{\mu_{2 \|}} \frac{\partial \varphi_{H}(b+0)}{\partial y} .
\end{gathered}
$$

Таким образом, определенные, согласно (6) и (7), операторы $\hat{T}_{E}, \hat{T}_{H}$ не являются самосопряженными в пространствах $L_{2}\left([0, w] \times[-c, c],\left|\varepsilon_{\perp}\left(1-\varepsilon_{\perp} \mu_{\|} \beta^{2}\right)\right|\right)$ и $L_{2}\left([0, w] \times[-c, c],\left|\mu_{\perp}\left(1-\varepsilon_{\|} \mu_{\perp} \beta^{2}\right)\right|\right)$, вследствие различия граничных условий для исходных и сопряженных операторов. Однако эти операторы подобны самосопряженным, т. е. существуют самосопряженный оператор $\hat{A}$ и ограниченный, ограниченно обратимый оператор $\hat{X}$, такие, что $\hat{T}=\hat{X}^{-1} \hat{A} \hat{X}$. В этом случае собственные функции операторов $\hat{T}_{E}, \hat{T}_{H}$ образуют базис Рисса, а вкупе с собственными функциями сопряженных задач они образуют электрическую и магнитную полные биортогональные системы [14]. При этом разложение произвольной функции из пространства $L_{2}([0, w] \times[-c, c] ;|\rho|)$ $(\rho-$ вес, $c-$ полуширина волновода в направлении неоднородности по диэлектрической и магнитной проницаемостям (рис. 1)) будет иметь вид $f=\sum_{n}\left\langle f, \Phi_{n}\right\rangle \Psi_{n}$.

Здесь $\langle\cdot, \cdot\rangle-$ скалярное произведение в пространстве $L_{2}([0, w] \times[-c, c] ;|\rho|), \Psi_{n}, \Phi_{n}-$ собственные функции поперечного оператора и сопряженного ему оператора соответственно. Таким образом, для поперечного электрического оператора выполнено

$$
\hat{T}_{E} \Psi_{E n}=\lambda_{E n} \Psi_{E n}, \quad \hat{T}_{E}^{*} \Phi_{E n}=\lambda_{E n} \Phi_{E n},
$$




$$
\left\langle\Phi_{E n}, \Psi_{E m}\right\rangle=\int\left|\varepsilon_{\perp}\left(1-\varepsilon_{\perp} \mu_{\|} \beta^{2}\right)\right| \Phi_{E n}^{*} \Psi_{E m} d x d y=\delta_{n, m} .
$$

Аналогично для поперечного магнитного оператора

$$
\hat{T}_{H} \Psi_{H n}=\lambda_{H n} \Psi_{H n}, \quad \hat{T}_{H}^{*} \Phi_{H n}=\lambda_{H n} \Phi_{H n},
$$

$\left\langle\Phi_{H n}, \Psi_{H m}\right\rangle=\int\left|\mu_{\perp}\left(1-\varepsilon_{\|} \mu_{\perp} \beta^{2}\right)\right| \Phi_{H n}^{*} \Psi_{H m} d x d y=\delta_{n, m}$.

При этом коэффициенты $A_{E}$ и $A_{H}$ в выражениях для $Y_{E_{y}}(y)$ и $Y_{H_{y}}(y)$ выбраны таким образом, чтобы собственные функции поперечных операторов были бы нормированными

$$
\begin{aligned}
\left\langle\Phi_{E n}, \Psi_{E n}\right\rangle & =\int\left|\varepsilon_{\perp}\left(1-\varepsilon_{\perp} \mu_{\|} \beta^{2}\right)\right| \Phi_{E n}^{*} \Psi_{E n} d x d y=1, \\
\left\langle\Phi_{H n}, \Psi_{H n}\right\rangle & =\int\left|\mu_{\perp}\left(1-\varepsilon_{\|} \mu_{\perp} \beta^{2}\right)\right| \Phi_{H n}^{*} \Psi_{H n} d x d y=1 .
\end{aligned}
$$

\section{Решения для нормальных к плоскости слоя компонент полей}

Будем искать решение в виде ряда по собственным функциям оператора $\hat{T}_{E}$

$$
E_{y}=\sum_{n, m} Z(\zeta) \Psi_{n, m}(x, y) .
$$

В дальнейшем для сокращения записи индексы суммирования $n, m$ будем опускать. Подставляя (22) в (8), получим

$\sum_{n, m} \Psi_{E}(x, y)\left(\frac{\partial^{2} Z}{\partial \xi^{2}}+\lambda_{E} Z\right)=\frac{-e}{\varepsilon_{0}\left(1-\varepsilon_{\perp} \mu_{\|} \beta^{2}\right)} \frac{\partial}{\partial y}\left(\frac{n}{\varepsilon_{\|}}\right)$.

Умножим обе части уравнения (23) на $\Phi_{E}^{*}(x, y)\left|\varepsilon_{\perp}\left(1-\varepsilon_{\perp} \mu_{\|} \beta^{2}\right)\right|$ и проинтегрируем по поперечному сечению волновода. Учитывая биортогональность (20) и выражение для концентрации заряда (3), получим

$$
\begin{gathered}
\int_{S} \Psi_{E}(x, y) \Phi_{E}^{*}(x, y)\left|\varepsilon_{\perp}\left(\varepsilon_{\perp} \mu_{\|} \beta^{2}-1\right)\right| d x d y\left(\frac{\partial^{2} Z}{\partial \xi^{2}}+\lambda_{E} Z\right) \\
=\frac{-q}{\varepsilon_{0}} \frac{\varepsilon_{1 \perp}}{\varepsilon_{1 \|}} \frac{\partial \Phi_{E}^{*}\left(x_{0} y_{0}\right)}{\partial y} \delta\left(\xi-z_{0}\right)
\end{gathered}
$$$$
\left(\frac{\partial^{2} Z}{\partial \xi^{2}}+\lambda_{E} Z\right)=\frac{-q}{\varepsilon_{0}\left\langle\Phi_{E} \Psi_{E}\right\rangle} \frac{\varepsilon_{1 \perp}}{\varepsilon_{1 \|}} \frac{\partial \Phi_{E}^{*}\left(x_{0}, y_{0}\right)}{\partial y} \delta\left(\xi-z_{0}\right) .
$$

Произведем преобразование Фурье по продольной координате $\xi$

$$
\left(-\kappa^{2}+\lambda_{E}\right) \mathscr{F}(Z)=\frac{-q \varepsilon_{1 \perp} \frac{\partial}{\partial y} \Phi_{E}^{*}\left(x_{0}, y_{0}\right)}{2 \pi \varepsilon_{0} \varepsilon_{1 \|}\left\langle\Phi_{E}, \Psi_{E}\right\rangle} \int_{-\infty}^{+\infty} e^{-i \kappa \xi} \delta\left(\xi-z_{0}\right) d \xi
$$

$$
\mathscr{F}(Z)=\frac{-q \varepsilon_{1 \perp} \frac{\partial}{\partial y} \Phi_{E}^{*}\left(x_{0}, y_{0}\right) e^{-i \kappa z_{0}}}{2 \pi \varepsilon_{0} \varepsilon_{1 \|}\left(-\kappa^{2}+\lambda_{E}\right)\left\langle\Phi_{E}, \Psi_{E}\right\rangle} .
$$

Применим обратное преобразование Фурье

$$
Z=\frac{-q \varepsilon_{1} \perp \frac{\partial}{\partial y} \Phi_{E}^{*}\left(x_{0}, y_{0}\right)}{2 \pi \varepsilon_{0} \varepsilon_{1 \|}\left\langle\Phi_{E}, \Psi_{E}\right\rangle} \int_{-\infty}^{+\infty} \frac{e^{-i \kappa z_{0}} e^{i \kappa \xi}}{\left(\lambda_{E}-\kappa^{2}\right)} d \kappa
$$

Беря последний интеграл, согласно теореме о вычетах, и учитывая (22), получим

$$
E_{y}=\sum_{n, m} \frac{q}{\varepsilon_{0}} \frac{\varepsilon_{1 \perp}}{\varepsilon_{1 \|}} \frac{\Psi_{E}(x, y)}{\left\langle\Phi_{E}, \Psi_{E}\right\rangle} \frac{\partial \Phi_{E}^{*}\left(x_{0}, y_{0}\right)}{\partial y} \frac{S_{E}(\xi)}{k_{z}}
$$

где $S_{E}(\xi)=-\sin \left(k_{z} \xi\right)$ при $\lambda_{E}>0, k_{z}=\sqrt{\lambda_{E}} ; S_{E}(\xi)=$ $=\exp \left(-k_{z} \xi\right)$ при $\lambda_{E}<0, k_{z}=\sqrt{\left|\lambda_{E}\right|}$.

Полученное решение при $\lambda_{E}>0$ представляет собой распространяющуюся внутри волновода волну с постоянной распространения $k_{z}$. Будем искать решение $(9)$ в виде ряда по собственным функциям оператора $\hat{T}_{H}$

$$
H_{y}=\sum_{n, m} Z(\zeta) \Psi_{H n, m}(x, y)
$$

$$
\sum_{n, m} \Psi_{H}(x, y)\left(\frac{\partial^{2} Z}{\partial \zeta^{2}}+\lambda_{H} Z\right)=\frac{-e v}{\left(1-\varepsilon_{\|} \mu_{\perp} \beta^{2}\right)} \frac{\partial n}{\partial x} .
$$

Умножим обе части (27) на $\left|\mu_{\perp}\left(1-\varepsilon_{\|} \mu_{\perp} \beta^{2}\right)\right| \Phi_{H}^{*}(x, y)$ и проинтегрируем по поперечному сечению волновода. Учитывая выражение (3) для концентрации электронов $n$ и биортогональность (21), получим

$$
\begin{aligned}
\int_{S} \Psi_{H}(x, y) \Phi_{H}^{*}(x, y)\left|\mu_{\perp}\left(1-\varepsilon_{\|} \mu_{\perp} \beta^{2}\right)\right| d x d y \\
\times\left(\frac{\partial^{2} Z}{\partial \xi^{2}}+\lambda_{H} Z\right)=-q v \mu_{1 \perp} \delta\left(\xi-z_{0}\right) \frac{\partial \Phi_{H}^{*}\left(x_{0}, y_{0}\right)}{\partial x} .
\end{aligned}
$$

Произведем преобразование Фурье по продольной координате $\xi$

$$
\left(-\kappa^{2}+\lambda_{H}\right) \mathscr{F}(Z)=\frac{-q v \mu_{1 \perp} e^{-i \kappa z_{0}}}{2 \pi\left\langle\Phi_{H}, \Psi_{H}\right\rangle} \frac{\partial \Phi_{H}^{*}\left(x_{0}, y_{0}\right)}{\partial x} .
$$

Применим обратное преобразование Фурье

$$
Z=\left.\frac{-q v \mu_{1 \perp}}{2 \pi\left\langle\Phi_{H}, \Psi_{H}\right\rangle}\left(\frac{\partial \Phi_{H}^{*}\left(x_{0}, y_{0}\right)}{\partial x}\right)\right|_{y=y_{0}} \int_{-\infty}^{+\infty} \frac{e^{i \kappa z_{0}} e^{-i \kappa \xi}}{\left(\lambda_{H}-\kappa^{2}\right)} d \kappa
$$

Беря последний интеграл, согласно теореме о вычетах, получим:

$$
H_{y}=q v \mu_{1 \perp} \sum_{n, m} \frac{\Psi_{H}(x, y)}{\left\langle\Phi_{H}, \Psi_{H}\right\rangle} \frac{\partial \Phi_{H}^{*}\left(x_{0} y_{0}\right)}{\partial x} \frac{S_{H}(\xi)}{k_{z}},
$$

где $S_{H}(\xi)=-\sin \left(k_{z} \xi\right)$ при $\lambda_{H}>0, k_{z}=\sqrt{\lambda_{H}}, S_{H}(\xi)=$ $=\exp \left(-k_{z} \xi\right) / 2$ при $\lambda_{H}<0, k_{z}=\sqrt{\left|\lambda_{H}\right|}$. Подставляя в 
полученные выражения собственные функции поперечных операторов и учитывая возможность отрицательных собственных чисел $\lambda$, получим

$$
\begin{aligned}
E_{y} & =\sum_{n, m} \frac{q}{\varepsilon_{0}} \sin \left(k_{x} x\right) \sin \left(k_{x} x_{0}\right) \frac{Y_{E_{y}}(y)}{\left\langle\Phi_{E}, \Psi_{E}\right\rangle} \frac{1}{\varepsilon_{1 \|}} \\
& \times \frac{\partial\left(\varepsilon_{1 \perp} Y_{E_{y}}^{*}\left(y_{0}\right)\right)}{\partial y} \frac{S_{E}\left(\zeta, z_{0}\right)}{\sqrt{\left|\lambda_{E}\right|}} \\
H_{y}=- & q v \sum_{n, m} \frac{k_{x} \cos \left(k_{x} x\right) \sin \left(k_{x} x_{0}\right) Y_{H_{y}}(y) \mu_{1 \perp} Y_{H_{y}}^{*}\left(y_{0}\right)}{\left\langle\Phi_{H}, \Psi_{H}\right\rangle} \\
\times & \frac{S_{H}\left(\xi, z_{0}\right)}{\sqrt{\left|\lambda_{H}\right|}}
\end{aligned}
$$

где

$$
\begin{aligned}
& S_{E, H}\left(\xi . z_{0}\right)= \\
& = \begin{cases}-\sin \left(\sqrt{\lambda_{E, H}}\left(\xi-z_{0}\right)\right) \theta\left(\xi-z_{0}\right) & \text { при } \\
\frac{\operatorname{sign}\left(\xi-z_{0}\right)}{2} \exp \left(-\sqrt{\left|\lambda_{E, H}\right|}\left|\xi-z_{0}\right|\right) & \lambda_{E, H}>0, \\
& \text { при } \\
& \lambda_{E, H}<0 .\end{cases}
\end{aligned}
$$

В случае изотропной среды $\varepsilon_{2 \perp}=\varepsilon_{2 \|}=\varepsilon_{2}$, $\mu_{2 \|}=\mu_{2 \perp}=\mu_{2}$ выражения для нормальных компонент полей $E_{y}, H_{y}$ совпадают с выражениями, полученными в $[14,17]$.

\section{Расчет тангенциальных к плоскости слоя компонент полей}

Необходимо отметить, что $\varepsilon(y)$ и $\mu(y)$, а также $E_{y}(y)$ и $H_{y}(y)$ не дифференцируемы на границе раздела слоев диэлектрического заполнения волновода, что делает ограниченно применимыми решения для тангенциальных компонент, полученные в [13]. Поэтому математически строгим подходом при выводе выражений для тангенциальных компонент полей является исключение операций дифференцирования по координате $y$ всех функций, которые скачкообразно меняются на границах раздела слоев. В $[14,17]$ для нахождения тангенциальных компонент полей использованы первообразные собственных функций поперечных операторов волновода. При этом в случае симметричных мод интегрирование по сечению волновода приводит к отличному от нуля значению тангенциальной составляющей поля на одной из границ. В связи с этим получим выражения через дифференциальные выражения, исключающие нарушения граничных условий.

Запишем первые два уравнения Максвелла (1) в декартовой системе координат

$$
\frac{\partial E_{z}}{\partial y}-\frac{\partial E_{y}}{\partial \xi}=v \frac{\partial\left(\mu_{0} \mu_{\|} H_{x}\right)}{\partial \xi}
$$

$$
\begin{gathered}
\frac{\partial E_{x}}{\partial \xi}-\frac{\partial E_{z}}{\partial x}=v \frac{\partial\left(\mu_{0} \mu_{\perp} H_{y}\right)}{\partial \xi}, \\
\frac{\partial E_{y}}{\partial x}-\frac{\partial E_{x}}{\partial y}=v \frac{\partial\left(\mu_{0} \mu_{\|} H_{z}\right)}{\partial \xi}, \\
\frac{\partial H_{z}}{\partial y}-\frac{\partial H_{y}}{\partial \xi}=-v \frac{\partial\left(\varepsilon_{0} \varepsilon_{\|} E_{x}\right)}{\partial \xi}, \\
\frac{\partial H_{x}}{\partial \xi}-\frac{\partial H_{z}}{\partial x}=-v \frac{\partial\left(\varepsilon_{0} \varepsilon_{\perp} E_{y}\right)}{\partial \xi}, \\
\frac{\partial H_{y}}{\partial x}-\frac{\partial H_{x}}{\partial y}=-v \frac{\partial\left(\varepsilon_{0} \varepsilon_{\|} H_{z}\right)}{\partial \xi}-e n v .
\end{gathered}
$$

Для нахождения $H_{z}$ продифференцируем (34) по $x$, (32) по $\xi$, просуммируем полученные выражения и подставим производные $\frac{\partial H_{x}}{\partial \xi}$ и $\frac{\partial E_{x}}{\partial \xi}$, выраженные из (30) и (31) соответственно

$$
\frac{\partial^{2} H_{z}}{\partial x^{2}}+\frac{\partial^{2} H_{z}}{\partial \xi^{2}}=-\frac{\partial}{\partial \xi}\left(\frac{1}{\mu_{\|}} \frac{\partial\left(\mu_{\perp} H_{y}\right)}{\partial y}\right)+v \varepsilon_{0} \varepsilon_{\perp} \frac{\partial}{\partial x} \frac{\partial E_{y}}{\partial \xi}
$$

Производя преобразование Фурье по продольной координате $\xi$, получим

$$
\begin{aligned}
& \frac{\partial^{2} \mathscr{F}\left(H_{z}\right)}{\partial x^{2}}-\kappa^{2} \mathscr{F}\left(H_{z}\right) \\
& =i \kappa\left[-\frac{1}{\mu_{\|}} \frac{\partial\left(\mu_{\perp} \mathscr{F}\left(H_{y}\right)\right)}{\partial y}+v \frac{\partial\left(\varepsilon_{0} \varepsilon_{\perp} \mathscr{F}\left(E_{y}\right)\right)}{\partial x}\right] .
\end{aligned}
$$

Учитывая, что $H_{z}$ непрерывно внутри волновода, будем искать решение в виде ряда

$$
H_{z}=\sum_{n, m=1}^{\infty} X_{H_{z} n}(x) Y_{H_{z} m}(y) Z_{H_{z}}(\zeta)
$$

Подставляя (38), (39) и (40) в (37) и приравнивая соответствующие члены рядов, ввиду линейной независимости системы гармонических функций получим

$$
\begin{aligned}
& \left(k_{x}^{2}+\kappa^{2}\right) X_{H_{z}} Y_{E_{z}} \mathscr{F}\left(Z_{E_{z}}\right) \\
& =-i \kappa\left[-\frac{1}{\mu_{\|}(y)} \frac{\partial\left(\mu_{\perp}(y) \mathscr{F}\left(H_{y}\right)\right)}{\partial y}+v \frac{\partial\left(\varepsilon_{0} \varepsilon_{\perp}(y) \mathscr{F}\left(E_{y}\right)\right)}{\partial x}\right] .
\end{aligned}
$$

Из (24) и (22), а также из (28) и (26) следует, что

$$
\begin{aligned}
& \mathscr{F}\left(E_{y, m}\right)= \\
& =\sum_{n, m=1}^{\infty} \frac{q \sin \left(k_{x} x\right) \sin \left(k_{x} x_{0}\right) Y_{E_{y}}(y) \frac{1}{\varepsilon_{1 \|}} \frac{\partial\left(\varepsilon_{1 \perp} Y_{E_{y}}^{*}\left(y_{0}\right)\right)}{\partial y} \exp \left(-i \kappa z_{0}\right)}{2 \pi \varepsilon_{0}\left(\kappa^{2}-\lambda_{E}\right)\left\langle\Phi_{E_{y}}, \Psi_{E_{y}}\right\rangle},
\end{aligned}
$$


$\mathscr{F}\left(H_{y, m}\right)=$

$=\sum_{n, m=1}^{\infty} \frac{q v \cos \left(k_{x} x\right) \sin \left(k_{x} x_{0}\right) Y_{H_{y}}(y)\left(-k_{x}\right) \mu_{1 \perp} Y_{H_{y}}^{*}\left(y_{0}\right) \exp \left(-\kappa z_{0}\right)}{2 \pi\left(\kappa^{2}-\lambda_{H}\right)\left\langle\Phi_{H_{y}}, \Psi_{H_{y}}\right\rangle}$,

где * относится к собственным функциям сопряженных операторов. Тогда

$$
\begin{aligned}
\mathscr{F}\left(H_{z}\right) & =\frac{-q v i \kappa e^{-i \kappa z_{0}} k_{x} X_{H_{z}}\left(x, x_{0}\right)}{2 \pi\left(k_{x}^{2}+\kappa^{2}\right)} \\
& \times\left[\frac{\varepsilon_{\perp} Y_{E_{y}}(y)}{\left(\kappa^{2}-\lambda_{E}\right)\left\langle\Phi_{E_{y}}, \Psi_{E_{y}}\right\rangle} \frac{\partial\left(\varepsilon_{1 \perp} Y_{E_{y}}^{*}\left(y_{0}\right)\right)}{\varepsilon_{\|} \partial y}\right. \\
& \left.+\frac{Y_{H_{y}}^{*}\left(y_{0}\right)}{\left(\kappa^{2}-\lambda_{H}\right)\left\langle\Phi_{H_{y}}, \Psi_{H_{y}}\right\rangle} \frac{\mu_{1 \perp} \partial\left(\mu_{\perp} Y_{H_{y}}(y)\right)}{\mu_{\|} \partial y}\right] .
\end{aligned}
$$

где $X_{H_{z}}\left(x, x_{0}\right)=\cos \left(k_{x} x\right) \sin \left(k_{x} x_{0}\right), k_{x}=n \pi / w$.

Применим обратное преобразование Фурье, полученный интеграл возьмем с использованием теоремы о вычетах

$$
\begin{aligned}
H_{z} & =-q v k_{x} \cos \left(k_{x} x\right) \sin \left(k_{x} x_{0}\right) \\
& \times\left[\frac{\left(G_{x}-G_{H}\right) \mu_{1 \perp} Y_{H_{y}}^{*}\left(y_{0}\right)}{\left(k_{x n}^{2}+\lambda_{H}\right)\left\langle\Phi_{H_{y}}, \Psi_{H_{y}}\right\rangle} \frac{\partial\left(\mu_{\perp}(y) Y_{H_{y}}(y)\right)}{\mu_{\|}(y) \partial y}\right. \\
& \left.+\frac{\left(G_{x}-G_{E}\right) \varepsilon_{\perp}(y) Y_{E_{y}}(y)}{\left(k_{x n}^{2}+\lambda_{E}\right)\left\langle\Phi_{E_{y}}, \Psi_{E_{y}}\right\rangle} \frac{\partial\left(\varepsilon_{1 \perp} Y_{E_{y}}^{*}\left(y_{0}\right)\right)}{\varepsilon_{1 \|} \partial y}\right] .
\end{aligned}
$$

Здесь $G_{x}\left(\xi, z_{0}\right)=\frac{\operatorname{sign}\left(\xi-z_{0}\right)}{2} e^{-k_{x}\left|\xi-z_{0}\right|}$,

$$
\begin{aligned}
& G_{E, H}\left(\xi, z_{0}\right)= \\
& = \begin{cases}\cos \left(\sqrt{\lambda_{E, H}}\left(\xi-z_{0}\right)\right) \theta\left(-\zeta+z_{0}\right) & \text { при } \lambda_{E, H} \geq 0, \\
\frac{\operatorname{sign}\left(\xi-z_{0}\right)}{2} e^{-\sqrt{\left|\lambda_{E, H}\right|}\left|\xi-z_{0}\right|} & \text { при } \lambda_{E, H}<0,\end{cases}
\end{aligned}
$$

$\theta(\xi)$ - функция Хевисайда.

Для нахождения $H_{x}$ продифференцируем (36) по $x$, подставим в него (34) и проинтегрируем полученное уравнение по $\xi$

$$
\frac{\partial^{2} H_{x}}{\partial x^{2}}+\frac{\partial^{2} H_{x}}{\partial \xi^{2}}=-\frac{\partial}{\partial x}\left(\frac{1}{\mu_{\|}} \frac{\partial\left(\mu_{\perp} H_{y}\right)}{\partial y}\right)-v \varepsilon_{0} \varepsilon_{\perp} \frac{\partial^{2} E_{y}}{\partial \xi^{2}} .
$$

Производя преобразование Фурье по продольной координате $\xi$ и используя (39) и (40), получим

$$
\begin{aligned}
\mathscr{F}\left(H_{x, m}\right) & =\frac{-q v \sin k_{x} x \sin k_{x} x_{0} e^{-i \kappa z_{0}}}{2 \pi\left(k_{x}^{2}+\kappa^{2}\right)} \\
& \times\left[\frac{\kappa^{2} \varepsilon_{\perp} Y_{E_{y}}(y)}{\left(\kappa^{2}-\lambda_{E}\right)\left\langle\Phi_{E_{y}}, \Psi_{E_{y}}\right\rangle} \frac{\partial\left(\varepsilon_{1 \perp} Y_{E_{y}}^{*}\left(y_{0}\right)\right)}{\varepsilon_{1 \|} \partial y}\right. \\
& \left.-\frac{\kappa_{x}^{2} \mu_{1 \perp} Y_{H_{y}}^{*}\left(y_{0}\right)}{\left(\kappa^{2}-\lambda_{H}\right)\left\langle\Phi_{H_{y}}, \Psi_{H_{y}}\right\rangle} \frac{\partial\left(\mu_{\perp} Y_{H_{y}}(y)\right)}{\mu_{\|} \partial y}\right] .
\end{aligned}
$$

Применим обратное преобразование Фурье, полученный интеграл возьмем с использованием теоремы о вычетах

$H_{x n, m}=q v \sin k_{x} x \sin k_{x} x_{0}$

$$
\begin{aligned}
& \times\left[\frac{k_{x}\left(G_{x} \sqrt{\left|\lambda_{H}\right|}-k_{x} S_{H}\right) \mu_{1 \perp} Y_{H_{y}}^{*}\left(y_{0}\right)}{\sqrt{\left|\lambda_{H}\right|}\left(k_{x n}^{2}+\lambda_{H}\right)\left\langle\Phi_{H_{y}}, \Psi_{H_{y}}\right\rangle} \frac{\partial\left(\mu_{\perp} Y_{H_{y}}(y)\right)}{\mu_{\|} \partial y}\right. \\
& \left.-\frac{\left(k_{x} G_{x} \sqrt{\left|\lambda_{E}\right|}+\lambda_{E} S_{E}\right) \varepsilon_{\perp}(y) Y_{E_{y}}(y)}{\sqrt{\left|\lambda_{E}\right|}\left(k_{x}^{2}+\lambda_{E}\right)\left\langle\Phi_{E_{y}}, \Psi_{E_{y}}\right\rangle} \frac{\partial\left(\varepsilon_{1 \perp} Y_{E_{y}}^{*}\left(y_{0}\right)\right)}{\varepsilon_{1 \|} \partial y}\right] .
\end{aligned}
$$

Для нахождения $E_{x}$ применим преобразование Фурье по продольной координате $\xi$ к (33) и подставим в него (41) и (40)

$$
\begin{aligned}
& \mathscr{F}\left(E_{x, m}\right)=\frac{q k_{x} \cos k_{x} x \sin k_{x} x_{0} e^{-i \kappa z_{0}}}{2 \pi \varepsilon_{0} \varepsilon_{\|}\left(k_{x}^{2}+\kappa^{2}\right)} \\
& \times\left[\frac{1}{\left(\kappa^{2}-\lambda_{E}\right)\left\langle\Phi_{E_{y}} \Psi_{E_{y}}\right\rangle} \frac{\partial\left(\varepsilon_{\perp} Y_{E_{y}}(y)\right)}{\partial y} \frac{\partial\left(\varepsilon_{1 \perp} Y_{E_{y}}^{*}\left(y_{0}\right)\right)}{\varepsilon_{1 \|} \partial y}\right. \\
& \left.+\frac{\left[\lambda_{H}\left(1-\varepsilon_{\|} \mu_{\perp} \beta^{2}\right)-\kappa^{2}\right] Y_{H_{y}}(y) \mu_{1 \perp} Y_{H_{y}}^{*}\left(y_{0}\right)}{\left(\kappa^{2}-\lambda_{H}\right)\left\langle\Phi_{H_{y}}, \Psi_{H_{y}}\right\rangle}\right] .
\end{aligned}
$$

Применим обратное преобразование Фурье, полученный интеграл возьмем с использованием теоремы о вычетах

$$
\begin{aligned}
& E_{x n, m}=\frac{-q \cos k_{x} x \sin k_{x} x_{0}}{\varepsilon_{0} \varepsilon_{\|}}\left[\frac{\left(G_{x} \sqrt{\left|\lambda_{E}\right|}-k_{x} S_{E}\right)}{\sqrt{\left|\lambda_{E}\right|}\left(k_{x}^{2}+\lambda_{E}\right)\left\langle\Phi_{E_{y}}, \Psi_{E_{y}}\right\rangle}\right. \\
& \times \frac{\partial\left(\varepsilon_{\perp}(y) Y_{E_{y}}(y)\right)}{\partial y} \frac{1}{\varepsilon_{1 \|}} \frac{\partial\left(\varepsilon_{1 \perp} Y_{E_{y}}^{*}\left(y_{0}\right)\right)}{\partial y} \\
& +\frac{\left[G_{x}\left(\lambda_{H}\left(1-\varepsilon_{\|} \mu_{\perp} \beta^{2}\right)+k_{x}^{2}\right) \sqrt{\left|\lambda_{H}\right|}+k_{x} \lambda_{H} \varepsilon_{\|} \mu_{\perp} \beta^{2} S_{H}\right]}{\sqrt{\left|\lambda_{H}\right|}\left(k_{x n}^{2}+\lambda_{H}\right)\left\langle\Phi_{H_{y}}, \Psi_{H_{y}}\right\rangle} \\
& \left.\times Y_{H_{y}}(y) \mu_{1 \perp} Y_{H_{y}}^{*}\left(y_{0}\right)\right] .
\end{aligned}
$$

Для нахождения $E_{z}$ подставим полученное выражение (45) в (31)

$$
\begin{aligned}
& \mathscr{F}\left(E_{z, n, m}\right)=\frac{q \sin k_{x} x_{0} i \kappa e^{-i \kappa z_{0}}}{2 \pi \varepsilon_{0} \varepsilon_{\|}\left(k_{x}^{2}+\kappa^{2}\right)} \\
& \times\left[\frac{\frac{\partial\left(\varepsilon_{\perp} Y_{E_{y}}(y)\right)}{\partial y} \frac{\varepsilon_{1 \perp}}{\varepsilon_{1 \|}} \frac{\partial}{\partial y} Y_{E_{y}}^{*}\left(y_{0}\right)}{\left(\kappa^{2}-\lambda_{E}\right)\left\langle\Phi_{E_{y}}, \Psi_{E_{y}}\right\rangle}\right. \\
& \left.-\frac{\left[\left(1-\varepsilon_{\|} \mu_{\perp} \beta^{2}\right)\left(\kappa^{2}-\lambda_{H}\right)-\varepsilon_{\|} \mu_{\perp} \beta^{2} k_{x}^{2}\right] Y_{H_{y}}(y) \mu_{1 \perp} Y_{H_{y}}^{*}\left(y_{0}\right)}{\left(\kappa^{2}-\lambda_{H}\right)\left\langle\Phi_{H_{y}}, \Psi_{H_{y}}\right\rangle}\right] .
\end{aligned}
$$


Применим обратное преобразование Фурье, полученный интеграл возьмем с использованием теоремы о вычетах

$$
\begin{aligned}
E_{z} & =\frac{q}{\varepsilon_{0}} \sum_{n, m}^{\infty} \sin k_{x} x \sin k_{x} x_{0}\left[\frac{\left(G_{x}-G_{E}\right)}{\left(k_{x n}^{2}+\lambda_{E}\right)\left\langle\Phi_{E_{y}}, \Psi_{E_{y}}\right\rangle} \frac{\partial\left(\varepsilon_{\perp}(y) Y_{E_{y}}(y)\right)}{\varepsilon_{\|}(y) \partial y} \frac{\partial\left(\varepsilon_{1 \perp} Y_{E_{y}}^{*}\left(y_{0}\right)\right)}{\varepsilon_{1 \|} \partial y}\right. \\
& \left.+\frac{\left[G_{x}\left[k_{x}^{2}+\left(1-\varepsilon_{\|}(y) \mu_{\perp}(y) \beta^{2}\right) \lambda_{H}\right]-\varepsilon_{\|}(y) \mu_{\perp}(y) \beta^{2} k_{x}^{2} G_{H}\right] Y_{H_{y}}(y) \mu_{1 \perp} Y_{H_{y}}^{*}\left(y_{0}\right)}{\varepsilon_{\|}(y)\left(k_{x}^{2}+\lambda_{H}\right)\left\langle\Phi_{H_{y}}, \Psi_{H_{y}}\right\rangle}\right] .
\end{aligned}
$$

Проверка полученных решений прямой подстановкой в систему уравнений Максвелла показала, что компоненты решения, содержащие $G_{x}$, им не удовлетворяют. В случае изотропной среды $\varepsilon_{2 \perp}=\varepsilon_{2 \|}=\varepsilon_{2}$, $\mu_{2 \|}=\mu_{2 \perp}=\mu_{2}$ выражение для продольной компоненты поля $E_{z}$ совпадает с выражениями, полученными в $[14,17]$.

\section{Компактная запись полученных решений}

Введем обозначения для компонент, связанных с электрическим полем:

$$
\begin{aligned}
& Y_{D_{y}}(y)=\varepsilon_{\perp}(y) Y_{E_{y}}(y)= \\
& =A_{E} \begin{cases}\cos \left(\left[k_{y 2 E}(c-y)\right),\right. & b \leq y \leq c, \\
{\left[\begin{array}{c}
\cosh \\
\sinh
\end{array}\right]\left(k_{y 1} y\right) \frac{\cos \left(k_{y 2 E}(c-b)\right)}{\left[\begin{array}{c}
\cosh \\
\sinh
\end{array}\right]\left(k_{y 1} b\right)},} & -b \leq y \leq b, \\
{[ \pm] \cos \left(k_{y 2 E}(c+y)\right),} & -c \leq y \leq-b .\end{cases} \\
& Y_{E_{-} d}(y)=\frac{\partial\left(\varepsilon_{\perp}(y) Y_{E_{y}}(y)\right)}{\varepsilon_{\|}(y) \partial y}= \\
& =A_{E} \begin{cases}\frac{k_{y 2 E}}{\varepsilon_{\| 2}} \sin \left(k_{y 2 E}(c-y)\right), & b \leq y \leq c, \\
k_{y 1}\left[\begin{array}{l}
\mathrm{sh} \\
\mathrm{ch}
\end{array}\right]\left(k_{y 1} y\right) \frac{\cos \left(k_{y 2 E}(c-b)\right)}{\varepsilon_{1}\left[\begin{array}{l}
\mathrm{ch} \\
\mathrm{sh}
\end{array}\right]\left(k_{y 1} b\right)}, & -b \leq y \leq b, \\
{\left[\begin{array}{c}
- \\
+
\end{array}\right] \frac{k_{y 2 E}}{\varepsilon_{\| 2}} \sin \left(k_{y 2 E}(c+y)\right),} & -c \leq y \leq-b .\end{cases} \\
& \begin{aligned}
Y_{E_{-} d}^{*}\left(y_{0}\right) & =\frac{\partial\left(\varepsilon_{1 \perp} Y_{E_{y}}^{*}\left(y_{0}\right)\right)}{\left\langle\Phi_{E}, \Psi_{E}\right\rangle \varepsilon_{1 \|} \partial y} \\
& =A_{E} k_{y 1} \frac{\cos \left(k_{y 2 E}(c-b)\right)}{\left[\begin{array}{c}
\mathrm{ch} \\
\mathrm{sh}
\end{array}\right]\left(k_{y 1} b\right)}\left[\begin{array}{c}
\mathrm{sh} \\
\mathrm{ch}
\end{array}\right]\left(k_{y 1} y_{0}\right) .
\end{aligned}
\end{aligned}
$$

Введем обозначения для компонент, связанных с магнитным полем:

$$
\begin{aligned}
& Y_{B_{y}}(y)=\mu_{\perp} Y_{H_{y}}(y)= \\
& =A_{H}\left\{\begin{array}{lc}
\sin \left(k_{y 2 H}(c-y)\right), & b \leq y \leq c \\
{\left[\begin{array}{l}
\mathrm{ch} \\
\mathrm{sh}
\end{array}\right]\left(k_{y 1} y\right) \frac{\sin \left(k_{y 2 H}(c-b)\right)}{\left[\begin{array}{l}
\mathrm{ch} \\
\mathrm{sh}
\end{array}\right]\left(k_{y 1} b\right)},} & -b \leq y \leq b, \\
{\left[\begin{array}{l}
+ \\
-
\end{array}\right] \sin \left(k_{y 2 H}(c+y)\right)} & -c \leq y \leq-b .
\end{array}\right.
\end{aligned}
$$$$
Y_{H \_d}(y)=\frac{1}{\mu_{\|}(y)} \frac{\partial\left(\mu_{\perp}(y) Y_{H_{y}}(y)\right)}{\partial y}=
$$$$
=A_{H}\left\{\begin{array}{lc}
-\frac{k_{y 2 H}}{\mu_{\| 2}} \cos \left(k_{y 2 H}(c-y)\right), & b \leq y \leq c \\
k_{y 1}\left[\begin{array}{l}
\mathrm{sh} \\
\mathrm{ch}
\end{array}\right]\left(k_{y 1} y\right) \frac{\sin \left(k_{y 2 H}(c-b)\right)}{\left[\begin{array}{l}
\mathrm{ch} \\
\mathrm{sh}
\end{array}\right]\left(k_{y 1} b\right)}, & -b \leq y \leq b, \\
{[+] \frac{k_{y 2 H}}{\mu_{\| 2}} \cos \left(k_{y 2 H}(c+y)\right),} & -c \leq y \leq-b .
\end{array}\right.
$$$$
Y_{B}^{*}\left(y_{0}\right)=\frac{\mu_{1 \perp} Y_{H_{y}}^{*}\left(y_{0}\right)}{\left\langle\Phi_{H}, \Psi_{H}\right\rangle}=A_{H} \frac{\sin \left(k_{y 2 H}(c-b)\right)}{\left[\begin{array}{l}
\mathrm{ch} \\
\mathrm{sh}
\end{array}\right]\left(k_{y 1} b\right)}\left[\begin{array}{l}
\mathrm{ch} \\
\mathrm{sh}
\end{array}\right]\left(k_{y 1} y_{0}\right) \text {. }
$$

Используя введенные обозначения, будем иметь:

$$
\begin{aligned}
E_{y}= & \sum_{n, m} \frac{q}{\varepsilon_{0}} \sin \left(k_{x} x\right) \sin \left(k_{x} x_{0}\right) Y_{E_{y}}(y) Y_{E_{-} d}^{*}\left(y_{0}\right) \frac{S_{E}\left(\xi, z_{0}\right)}{\sqrt{\left|\lambda_{E}\right|}}, \\
E_{z}= & -\frac{q}{\varepsilon_{0}} \sum_{n, m}^{\infty} \sin k_{x} x \sin k_{x} x_{0} \\
& \times\left[\frac{G_{E} Y_{E_{-} d}(y) Y_{E_{-} d}^{*}\left(y_{0}\right)}{\left(k_{x}^{2}+\lambda_{E}\right)}+\frac{k_{x}^{2} \beta^{2} G_{H} Y_{B_{y}}(y) Y_{B}^{*}\left(y_{0}\right)}{\left(k_{x}^{2}+\lambda_{H}\right)}\right], \\
E_{x}= & \frac{q}{\varepsilon_{0}} \sum_{n, m}^{\infty} \cos k_{x} x \sin k_{x} x_{0} \\
\times & {\left[\frac{k_{x} S_{E} Y_{E_{-} d}(y) Y_{E_{-} d}^{*}\left(y_{0}\right)}{\sqrt{\left|\lambda_{E}\right|}\left(k_{x}^{2}+\lambda_{E}\right)}-\frac{k_{x} \lambda_{H} S_{H} \beta^{2}}{\sqrt{\left|\lambda_{H}\right|}\left(k_{x}^{2}+\lambda_{H}\right)} Y_{B_{y}}(y) Y_{B}^{*}\left(y_{0}\right)\right], }
\end{aligned}
$$




$$
\begin{aligned}
H_{y}=- & q v \sum_{n, m} k_{x} \cos \left(k_{x} x\right) \sin \left(k_{x} x_{0}\right) Y_{H_{y}}(y) Y_{B}^{*}\left(y_{0}\right) \frac{S_{H}\left(\xi, z_{0}\right)}{\sqrt{\left|\lambda_{H}\right|}}, \\
H_{z}= & q v \sum_{n, m} k_{x} \cos \left(k_{x} x\right) \sin \left(k_{x} x_{0}\right) \\
& \times\left[\frac{G_{H} Y_{H_{-} d}(y) Y_{B}^{*}\left(y_{0}\right)}{\left(k_{x}^{2}+\lambda_{H}\right)}+\frac{G_{E} Y_{D_{y}}(y) Y_{E \_d}^{*}\left(y_{0}\right)}{\left(k_{x}^{2}+\lambda_{E}\right)}\right], \\
H_{x}= & q v \sum_{n, m} \sin k_{x} x \sin k_{x} x_{0} \\
& \times\left[\frac{k_{x}^{2} S_{H} Y_{H_{\_} d}(y) Y_{B}^{*}\left(y_{0}\right)}{\sqrt{\left|\lambda_{H}\right|}\left(k_{x}^{2}+\lambda_{H}\right)}-\frac{\lambda_{E} S_{E} Y_{D_{y}} Y_{E \_d}^{*}\left(y_{0}\right)}{\sqrt{\left|\lambda_{E}\right|}\left(k_{x}^{2}+\lambda_{E}\right)}\right] .
\end{aligned}
$$

Поперечные силы, действующие на электроны внутри волновода, могут быть найдены с использованием формулы Лоренца $F_{x}=-e\left(E_{x}-v B\right), F_{y}=-e\left(E_{y}+v B_{x}\right)$ :

$$
\begin{aligned}
F_{x} & =-\frac{q e}{\varepsilon_{0}} \sum_{n, m}^{\infty} k_{x} \cos k_{x} x \sin k_{x} x_{0} \\
& \times\left[\frac{S_{E} Y_{E \_d}(y) Y_{E_{-} d}^{*}\left(y_{0}\right)}{\sqrt{\left|\lambda_{E}\right|}\left(k_{x}^{2}+\lambda_{E}\right)}+\frac{S_{H} k_{x}^{2} \beta^{2} Y_{B_{y}}(y) Y_{B}^{*}\left(y_{0}\right)}{\sqrt{\left|\lambda_{H}\right|}\left(k_{x}^{2}+\lambda_{E}\right)}\right], \\
F_{y} & =-\frac{q e}{\varepsilon_{0}} \sum_{n, m} \sin \left(k_{x} x\right) \sin \left(k_{x} x_{0}\right) \\
& \times\left[\left(\frac{S_{E}}{\sqrt{\left|\lambda_{E}\right|}}\left(\frac{1}{\varepsilon_{\perp}}-\frac{\mu_{\|} \lambda_{E} \beta^{2}}{k_{x}^{2}+\lambda_{E}}\right)\right) Y_{D_{y}}(y) Y_{E_{-} d}^{*}\left(y_{0}\right)\right. \\
& \left.+\frac{k_{x}^{2} \beta^{2} \mu_{\|} S_{H} Y_{H_{-} d}(y) Y_{B}^{*}\left(y_{0}\right)}{\sqrt{\left|\lambda_{H}\right|}\left(k_{x}^{2}+\lambda_{H}\right)}\right] .
\end{aligned}
$$

\section{Расчет электромагнитных полей ускоряющих структур прямоугольного сечения \\ с трансверсально-анизотропным заполнением}

На основе полученных выражений был проведен аналитический анализ кильватерных полей, генерируемых гауссовым релятивистским электронным сгустком с параметрами ускорителя AWA в прямоугольной ускоряющей структуре на основе сапфиpa [4]: $w=11 \mathrm{~mm}, b=1.5 \mathrm{~mm}, c=2.39 \mathrm{~mm}, \varepsilon_{2 \perp}=11.5$,

Базовая частота волноводов с заполнением от диэлектрической проницаемости

\begin{tabular}{c|c|c|c|c}
\hline $\begin{array}{c}\text { Диэлектрическая } \\
\text { проницаемость }\end{array}$ & $\begin{array}{c}\varepsilon_{2 \perp}=11.5, \\
\varepsilon_{2 \|}=9.4\end{array}$ & $\varepsilon_{2}=11.5$ & $\varepsilon_{2}=10.45$ & $\varepsilon_{2}=9.4$ \\
\hline $\begin{array}{c}\text { Базовая частота, } \\
\mathrm{GHz}\end{array}$ & 25 & 23.25 & 24.23 & 25.36
\end{tabular}

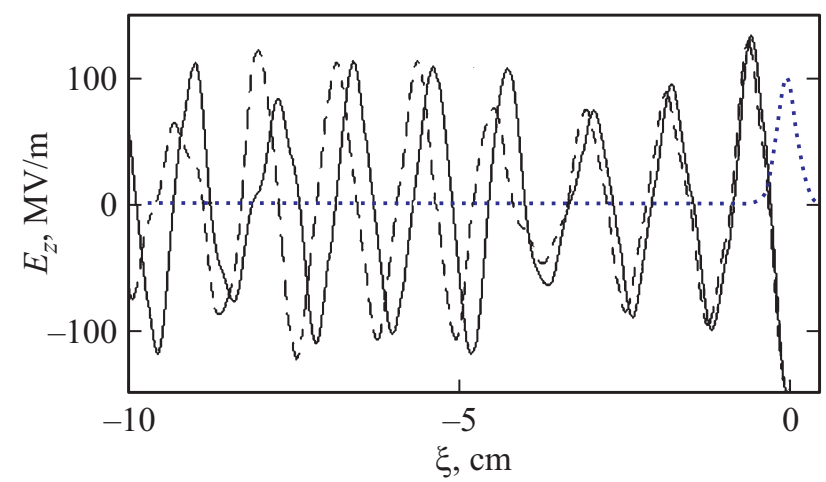

Рис. 2. Зависимость продольного электрического поля в прямоугольной ускоряющей структуре на основе сапфира.

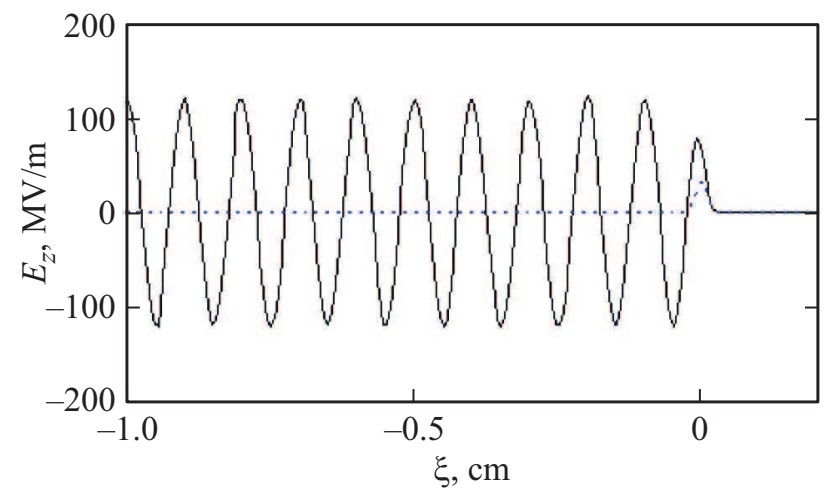

Рис. 3. Зависимость продольного электрического поля для субтерагерцовой прямоугольной ускоряющей структуры на основе сапфира.

$\varepsilon_{2 \|}=9.4, \mu_{2 \|}=\mu_{2 \perp}=1$. Указанные параметры соответствуют частоте $25 \mathrm{GHz}$ ускоряющей LM-моды структуры. В таблице приведено сопоставление базовой частоты волноводов с приведенными выше геометрическими размерами, но заполненных изотропным диэлектриком со значениями диэлектрических проницаемостей, равными поперечной, продольной проницаемостям и среднему арифметическому из них. Видно, что частота низшей LM-моды с учетом анизотропии оказалась ближе к частоте, которая была бы при использовании компоненты $\varepsilon_{2}=\varepsilon_{2 \|}=9.4$.

В качестве источника черенковского излучения рассматривался генераторный электронный сгусток с гауссовым распределением заряда и энергией $W=15 \mathrm{MeV}$, зарядом $q=100 \mathrm{nC}$ и длиной сгустка $\sigma_{z}=1.5 \mathrm{~mm}$. Зависимость продольной компоненты электрического поля $E_{z}$, создаваемого сгустком, от расстояния за ним $\xi=z-v t$ представлена на рис. 2 (сгусток расположен в точке $x_{0}=w / 2, y_{0}=0, \xi_{0}=8 \mathrm{~cm}$; точка наблюдения $x=w / 2, y=0, \xi=z-v t)$. Сплошная линия соответствует расчету для сапфира с учетом анизотропии заполнения, прерывистая - расчету для случая изотропного заполнения с $\varepsilon_{2}=10.45$. Учет анизотропии приводит 

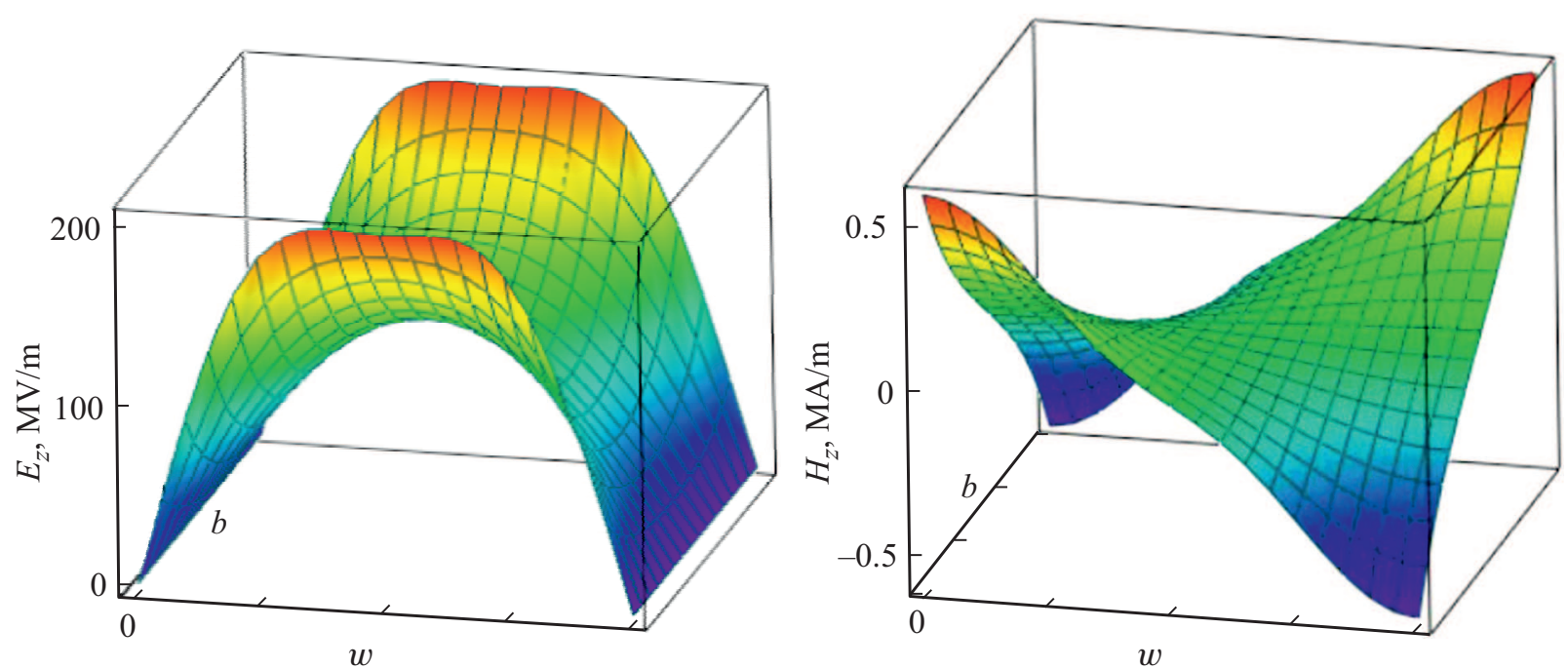

Pис. 4. Поперечная структура полей $E_{z}$ и $H_{z}$.

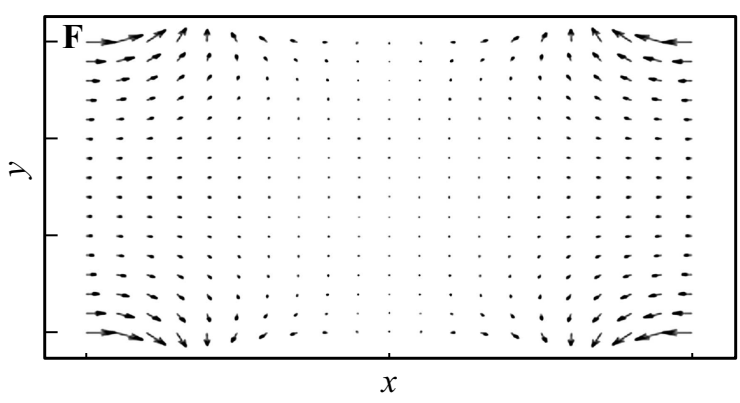

Рис. 5. Структура поперечных сил.

как к частотному сдвигу возбуждения структуры, так и изменению амплитуды поля.

На рис. 3 представлены зависимости продольной компоненты электрического поля для субтерагерцовой ускорительной структуры с параметрами $w=2.5 \mathrm{~mm}$, $b=1.0 \mathrm{~mm}, c=1.04 \mathrm{~mm}, \varepsilon_{2 \perp}=11.5, \varepsilon_{2 \|}=9.4$. При этом базовая частота волновода составляет $300 \mathrm{GHz}$. Генераторный сгусток имеет гауссово распределение заряда, энергию $W=75 \mathrm{MeV}$, заряд $q=10 \mathrm{nC}$ и длину $\sigma_{z}=0.1 \mathrm{~mm}$. Сгусток расположен в точке $x_{0}=w / 2$, $y_{0}=0, \xi_{0}=0 \mathrm{~cm}$; точка наблюдения $x=w / 2, y=0$, $\xi=z-v t$. Отметим, что при возбуждении указанным пучком обеспечивается высокий (более $100 \mathrm{MV} / \mathrm{m}$ ) ускоряющий градиент кильватерного излучения за сгустком в сочетании с практически одномодовым режимом возбуждения.

На рис. 4 представлены зависимости поперечной структуры полей $E_{z}$ и $H_{z}$ в точке, соответствующей первому за сгустком максимуму продольного электрического поля. Максимальное значение продольного электрического поля достигается вблизи границы диэлектрического слоя и оказывается примерно вдвое выше, чем поле в центральной части. Магнитное поле вблизи оси волновода, возбуждаемого осевым электронным пучком, близко к нулю, при этом необходимо отметить возникновение значительных продольных магнитных полей вблизи углов, порождающих значительные магнитные потери энергии в волноводе.

На рис. 5 представлена векторная структура распределения поперечных сил по сечению волновода. В направлении, перпендикулярном слоям, силы, действующие на частицы, направлены от оси волновода в направлении, параллельном слоям, силы притягивают частицы к оси аналогично полям жесткой фокусировки FODO. Это дает возможность использовать скрещенные под углом $90^{\circ}$ прямоугольные структуры для дополнительной фокусировки хвостовой части пучка [7]. Анализ фокусирующей системы применительно к прямоугольному волноводу выполнен в $[18,19]$.

\section{Заключение}

Представленый строгий аналитический метод расчета спектра и кильватерных полей излучения Вавилова-Черенкова в диэлектрической ускорительной структуре прямоугольного сечения на основе разложения решений системы уравнений Максвелла по собственным функциям поперечных операторов применим как к структурам с изотропным, так и трансверсальноизотропным заполнением. Полученные выражения для тангенциальных к диэлектрическому слою компонент полей приведены к удобной для расчетов форме. Использование аналитических выражений делает расчет полей независящим от частотного диапазона и геометрических размеров структуры. Аналитический подход также позволяет проводить существенно более быстрый и эффективный расчет как полей, так и самосогласованного движения заряженного пучка в генерируемых им полях по 
сравнению с программами, использующими численное моделирование полей стандартными методами.

\section{Конфликт интересов}

Авторы заявляют, что у них нет конфликта интересов.

\section{Список литературы}

[1] Gai W., Schoessow P., Cole B., Konecny R. et al. // Phys. Rev. Lett. 1988. Vol. 61. P. 2756.

[2] Gai W. // AIP Conf. Proceed. 2009. Vol. 1086. P. 3-11.

[3] Альтмарк А.М., Канарейкин А.Д., Шейнман И.Л. // ЖТФ. 2005. Т. 75. Вып. 1. С. 89-97.

[4] Kanareykin A. // J. Phys. Conf. Ser. 2010. Vol. 236. P. 012032.

[5] Rosing M., Gai W. // Phys. Rev. D. 1990. Vol. 42. N 5. P. $1829-1834$.

[6] Канарейкин А.Д., Шейнман И.Л. // Письма в ЖТФ. 2007. Т. 33. Вып. 8. С. 59-64.

[7] Xiao L., Gai W., Sun X. // Phys. Rev. E. 2001. Vol. 65. P. 1-9.

[8] Jing C., Liu W., Gai W., Xiao L., Wong T. // Phys. Rev. E. 2003. Vol. 68. P. 016502.

[9] Tremaine A., Rosenzweig J., Schoessow P., Gai W. // Phys. Rev. E. 1997. Vol. 56. P. 7204.

[10] Wang C., Hirshfield J.L. // Phys. Rev. ST Accel. Beams. 2006. Vol. 9. N 3. P. 031301(18).

[11] Balanis C.A. Advanced Engineering Electromagnetics. NY: Wiley, 1989. P. 410-414.

[12] Сотников Г.В., Онищенко И.Н., Хирибилд Джс.Л., Маршалл T.K. // Проблемы атомной науки и технологии. 2008. № 3. Серия: Ядерно-физические исследования (49). C. $148-152$.

[13] Батурин С.С., Шейнман И.Л., Альтмарк А.М., Канарейкин А.Д. // ЖТФ. 2012. Т. 82. Вып. 5. С. 106-114

[14] Baturin S.S., Sheinman I.L., Altmark A.M., Kanareykin A.D. // Phys. Rev. ST Accel. Beams. 2013. Vol. 16. P. 051302 .

[15] Sheinman I., Baturin S., Kanareykin A. Analysis of an Rectangular Dielectric-lined Accelerating Structure with an Anisotropic Loading IPAC'12, New Orlean, USA, 2012. P. 2769-2771.

[16] Sheinman I., Sheinman Yu. Wake Field Components in a Rectangular Accelerating Structure with Dielectric Anisotropic Loading. RUPAC-2016, TUPSA043, 21-25 November 2016, Saint-Petersburg, Russia P. 310-312.

[17] Altmark A.M., Baturin S.S., Sheinman I.L., Kanareykin A.D. // J. Phys. Conf. Series. 2014. Vol. 517. N 1. P. 012005.

[18] Шейнман И.Л., Толстой А.Л. Динамика генераторного сгустка, фокусируемого путем переориентации прямоугольного диэлектрического волновода. Электроника и микроэлектроника СВЧ. 2018. № 1 (1). С. 633-637.

[19] Sheinman I.L., Tolstoy A.L. Driver Beam Stabilization in Rectangular Dielectric Waveguide. 2018 IEEE Conference of Russian Young Researchers in Electrical and Electronic Engineering (ElConRus), January 29-February 01, 2018. Moscow and St. Petersburg, Russia. P. 509-511. 